\title{
PERICULO PROCURATIO: MANAGMENT RISK
}

\author{
Dott. Quarticelli Antonio
}

TSRM professionista in possesso di Master di $1^{\circ}$

livello in management per le funzioni di Coordinamento.

KEYWORDS: Management Risk, Servizio di Radiologia, Diagnostica per Immagini

\section{ABSTRACT}

The Management Risk originated in the early 1980s in the United States with the aim of reducing the tendency to legal recourse by patients who had unforeseen clinical incidents that occurred during health treatments to which they were subjected. Also in Italy the Management Risk is born and develops to protect the employees of the National Health System and to protect the health of Patients / Users. The study will examine in a practical way the medical and legal responsibilities of a Medical Radiology Technician in the protection of the patient / client.

\section{INTRODUZIONE}

Lingua Latina:Periculo: pericolo/rischio; Procuratio: responsabilità.

Lingua anglosassone: Management: gestione; Risk: rischio.

Il sinonimo coniato dagli antichi Latini rende effettivamente meglio il significato del sinonimo in lingua Anglosassone; comunque, patriottismi a parte, il Management Risk ha origine nei primi anni 1980 negli Stati Uniti con lo scopo di ridurre la tendenza ai ricorsi legali da parte dei pazienti che hanno avuto incidenti clinici non previsti verificatisi durante trattamenti sanitari a cui sono stati sottoposti. Successivamente anche in Inghilterra, studi eseguiti dal National Health Service, indicano che in Gran Bretagna statisticamente nell' anno 2000 ci sono stati circa 400 pazienti deceduti conseguentemente a malfunzionamenti di apparecchiature mediche ,10 mila pazienti per danni da farmaci e 30 mila denunce per errori medici e paramedici per svariate tipologie:

\section{Errori Organizzativi \\ 2. Errori diagnostici \\ 3. Errori farmacologici \\ 4. Fallimento nel prevenire lesioni \\ 5. Errori interventistici \\ 6. Errori tecnici}

Anche in Italia il Management Risk nasce e si sviluppa per tutelare i dipendenti del sistema Sanitario Nazionale e per tutelare salute dei Pazienti/Utenti. Precisate le origini, le cause e la funzione dello studio del Management Risk, cerchiamo in maniera semplice di poter estrapolare il concetto. In questi anni si sono sviluppate, a livello Universitario molte figure di categoria paramedica specializzate in campo forense: viene subito da chiedersi "cui prodest?" (a chi giova) e a quale interesse queste specializzazioni vengono conseguite? Lo studio esaminerà in maniera pratica le responsabilità medico legali di un Tecnico Sanitario di Radiologia Medica nella tutela del paziente/cliente. Definizione di Rischio Clinico: Il rischio clinico è la probabilità che un paziente sia vittima i un evento avverso, cioè subisca qualsiasi danno o disagio imputabile, anche se in modo involontario alle cure mediche prestate durante il periodo di degenza, che causa un prolungamento del periodo di degenza un peggioramento delle condizioni di salute o la morte ed un aumento della spesa economica.

\section{PERICULO PROCURATIO}

Prima di affrontare l'argomento complesso del management risk ritengo necessario specificare la figura professionale e giuridica del Tecnico Sanitario di Radiologia Medica.

Il Parlamento Italiano legifera con pubblicazione nella Gazzetta Ufficiale n.208 del 6 settembre 2000 la disciplina delle professioni infermieristiche, tecniche, della riabilitazione, della prevenzione nonché della professione ostetrica.

Tale legge decreta:

- $\quad$ art.1 "I Tecnici Sanitari di Radiologia (operatori delle professioni sanitarie) svolgono con autonomia Professionale attività dirette alla cura, alla prevenzione e alla salvaguardia della salute individuale e collettiva, espletando le funzioni individuate dalla norma istituita dai relativi profili professionali, nonché degli specifici codici deontologici ed utilizzando metodologie di pianificazione per obbiettivi dell'assistenza."

- art.3 "Gli operatori delle professioni sanitarie dell'area tecnico-diagnostica svolgono, con autonomia professionale, le procedure tecniche necessarie alla esecuzione di metodiche diagnostiche su materiali biologici o sulla persona, ovvero attività tecnico- assistenziale, in attuazione di quanto previsto nei regolamenti concernenti l'individuazione delle figure e dei relativi profili professionali definiti con decreto del Ministro della sanità. Lo Stato e le regioni promuovono ,nell'esercizio delle proprie funzioni legislative, di indirizzo, di programmazione ed amministrative, lo sviluppo e la valorizzazione delle funzioni delle professioni sanitarie dell'area tecnicosanitaria, al fine di contribuire, anche attraverso la diretta responsabilizzazione di funzioni organizzative e didattiche, al diritto alla salute del cittadino, al processo di aziendalizzazione e al miglioramento della qualità organizzativa e professionale nel Servizio sanitario nazionale con l'obiettivo di una integrazione omogenea con i servizi sanitari e gli ordinamenti degli altri Stati dell'Unione europea."

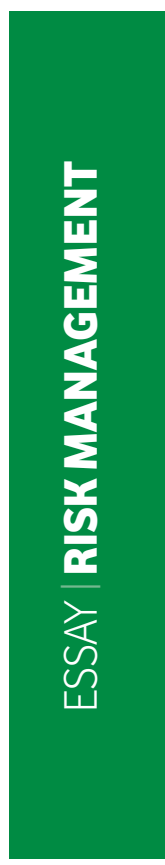


- $\quad$ art.5 "La formazione di tali figure professionali è di competenza del ministro dell'Università e della ricerca scientifica e tecnologica, di concerto con il Ministro della sanità."

Successivamente il Parlamento Italiano legifera il 1 febbraio 2006 la n.43 contenente le disposizioni in materia di professioni sanitarie infermieristiche, ostetrica, riabilitazione, tecnico-sanitarie e della prevenzione e delega al Governo l'istituzione dei relativi ordini professionali.

Si evince che la legge $n .43$ rimarca la legge $n .251$ del 10 agosto 2000 in merito a definizione(art.1) e requisiti(art.2), muta l'albo professionale in ordine delle professioni sanitarie definendo, finalmente (art.6 della legge 43/2006) una articolazione delle professioni sanitarie come di seguito riportata (anteriormente all'attivazione dei corsi di laurea o di diploma a esso equipollente ai sensi dell'art.4della legge 26 febbraio 1992, n.42):

1. professionisti in possesso del diploma di laurea o del titolo universitario conseguito anteriormente all'attivazione dei corsi di laurea o di diploma a esso equipollente ai sensi dell'art.4della legge 26 febbraio 1992, n.42;

2. professionisti coordinatori in possesso di master di primo livello in management o per le funzioni di coordinamento rilasciato dall'università ai sensi dell'art.3, comma 8, del regolamento di cui al Decreto del Ministro dell'Università e della ricerca scientifica e tecnologica 3 novembre 199, n.509 e dell'art.3, comma 9, del regolamento di cui al Decreto del Ministro dell'Istruzione, dell'Università e della ricerca22 ottobre 2004, n.270;

3. professionisti specialisti in possesso del master di primo livello per le funzioni specialistiche rilasciato dall'università ai sensi dell'art.3, comma 8, del regolamento di cui al Decreto del Ministro dell'Università e della ricerca scientifica e tecnologica 3 novembre 199, n.509 e dell'art.3, comma 9, del regolamento di cui al Decreto del Ministro dell'Istruzione, dell'Università e della ricerca22 ottobre 2004, n.270;

4. professionisti dirigenti in possesso di laurea specialistica di cui al Decreto del Ministro dell'Università e della ricerca scientifica e tecnologica 2 aprile 2001, e che abbiano esercitato l'attività professionale con rapporto di lavoro dipendente per almeno 5 anni, oppure ai quali siano conferiti incarichi dirigenziali ai sensi dell'art.7 della legge 10 agosto n.251.

Per terminare con la legge del 30 dicembre 2010, $n^{\circ}$ 240, all'art 17(equipollenze).

"I diplomi delle scuole dirette a fini speciali istituite ai sensi del decreto del Presidente della Repubblica 10 marzo $1982, \mathrm{n}^{\circ} 162$, riconosciuti al termine di un corso di durata triennale, e i diplomi universitari istituiti ai sensi della legge 19 novembre 1990, n³41, purché della medesima durata, sono equipollenti alle lauree di cui all'art.3, comma 1, lettera a del regolamento di cui al decreto del Ministro dell'Università e della Ricerca Scientifica e Tecnologia del 3 novembre 1999, n509.

Ai diplomati di cui comma 1 compete la qualifica accademica di "dottore" prevista per i laureati di cui all'art.13, comma 7, del decreto del Ministero dell'Istruzione. Ma senza dimenticare le nostre origini di Tecnici Sanitari di Radiologia Medica di conseguenza la nostra qualifica è prima di tutto di T.S.R.M. e poi a seguire la qualifica accademica di "dottore". Con ciò si definisce quindi che le professioni sanitarie oggi hanno definitivamente superato il concetto di ausiliaria tipico degli anni scorsi, assumendo una diretta responsabilità delle proprie attività.

La responsabilità del Tecnico Sanitario di Radiologia Medica consiste nelle conseguenze penali e o patrimoniali a cui va incontro il soggetto che assume un comportamento attivo(azione) o passivo(omissione) lesivo di un interesse tutelato dalla legge.

Con l'art.28 della Costituzione Italiana, vengono quindi precisati i soggetti e i profili di responsabilità; la responsabilità diretta del soggetto pubblico e l'estensione delle responsabilità allo Stato e agli Enti Pubblici.

Sui profili di responsabilità la norma costituzionale afferma le responsabilità di tipo penale, civile, amministrativo-disciplinare. Definita la parte giuridica e professionale di un Tecnico Sanitario di Radiologia Medica ad oggi, si passa ad esaminare la parte pratica del Management Risk.

\section{LA PRESTAZIONE RADIOLOGICA}

La prestazione di Diagnostica per Immagini comprende un insieme complesso ed articolato di prestazioni competenze, conoscenze e modus operandi di più professioni(attori), non solo dell'ambito medico. Il suo svolgimento si inserisce nell'ambito delle prestazioni sanitarie "d'equipe". La prestazione Diagnostica per Immagini, intesa come prestazione professionale con finalità diagnostiche, costa di una serie di momenti strettamente interdipendenti ed inscindibili. Esso è l'insieme di una serie di processi che possono essere riassunti in 7 fasi distinte.

1. Valutazione della richiesta di prestazione del medico proscrittore.

2. Inquadramento clinico-laboratoristico-anamnestico.

3. Giustificazione da parte del Medico Radiologo della prestazione proposta.

4. Esecuzione della prestazione.

5. Interpretazione/refertazione/comunicazione.

6. Archiviazione.

7. Conservazione.

Ogni richiesta ed esecuzione di prestazione di Diagnostica per Immagini deve sempre concludersi con un referto scritto di competenza del Medico Radiologo il quale esprime la sua valutazione di medico specialista, sulla base della semeiotica della propria specialità, al quesito posto dal clinico proponente.

Il Tecnico Sanitario di Radiologia Medica è l'unico interlocutore qualificato competente ed autorizzato ex lege, nei confronti anche degli altri medici specialisti che utilizzano in modo complementare la radiodiagnostica e come professionista di collegamento anche con il responsabile dell'impianto radiologico, lo specialista dell'area radiologica e l'esperto di fisica medica.

I mezzi di contrasto, farmaci a tutti gli effetti, non devono essere somministrati in assenza di una chiara indicazione clinica, raccolta di relativo consenso in- 
formato e adeguata valutazione clinica-anamnesticalaboratoristica del paziente da parte del Medico Radiologo.

Le problematiche legate al principio di giustificazione, raccolta dei dati clinico-anamnestici, informativa e consenso alla prestazione di Diagnostica per Immagini, sono differenziate a seconda che si tratti di prestazioni rese in regime di ricovero, in UrgenzaEmergenza o in Elezione, o in regime Ambulatoriale. Responsabilità Professionale

Attitudine ad essere chiamati a rispondere delle proprie azioni (ruolo, competenza, capacità, autonomia, etica, deontologia), obbligo di sopportare le conseguenze di un comportamento che abbia cagionato ad altri un danno.

Responsabilità penale (personale)

Reato (lesioni personali, omicidio), causalità (al di là di ogni ragionevole dubbio), dimostrazione della responsabilità a carico dell'accusato. Colpa generica per imperizia, imprudenza, negligenza. Colpa specifica per inosservanza di leggi, regolamenti, ordini o discipline.

\section{RESPONSABILITÀ CIVILE}

Trasferibile (assicurazione), danno ingiusto risarcibile, casualità, onere della prova diversificato (contrattuale vs extracontrattuale)

Extra-contrattuale: qualunque fatto doloso o colposo, che cagiona ad altri un danno ingiusto,obbliga colui che ha commesso il fatto a risarcirne il danno.

Contrattuale: Il Professionista che non esegue esattamente la prestazione dovuta è tenuto al risarcimento del danno, se non prova che l'inadempimento è stato determinato da impossibilità della prestazione derivante da causa a lui non imputabile.

Responsabilità amministrativa: il Professionista risponde per dolo o per colpa grave per i danni all'erario causati nell'ambito del rapporto di lavoro (art.28 della Costituzione Italiana)

\section{DECRETO BALDUZZI}

art.3 DL 158/12 Stabilisce che l'esercente della professione sanitaria che nello svolgimento della propria attività si attiene alle linee guida e alle buone pratiche accreditate dalla comunità scientifica non risponde penalmente per colpa lieve. In tali casi resta comunque fermo l'obbligo di cui all'art.2043 del codice civile "risarcimento del danno": qualunque fatto Doloso o Colposo, che cagiona ad altri un danno ingiusto, obbliga colui che ha commesso il fatto a risarcire il danno. Il giudice, anche nella determinazione del risarcimento del danno, tiene debitamente conto della condotta di cui al primo periodo.

\section{LEGGE GELLI}

Legge del 17/03/2017 stabilisce le disposizioni in materia di sicurezza delle cure e della persona assistita nonché in materia di responsabilità professionale degli esercenti le professioni sanitarie.

- $\quad$ art.1 "La sicurezza delle cure, è parte costitutiva del diritto alla salute ed è perseguita nell'interesse dell'individuo e della collettività.La sicurezza delle cure si realizza anche mediante l'insieme di tutte le attività finalizzate alla prevenzione e alla gestione del rischio connesso all'erogazione di prestazioni sanitarie e l'utilizzo appropriato del- le risorse strutturali, tecnologiche e organizzative. Alle attività di prevenzione del rischio messe in atto dalle strutture sanitarie e sociosanitarie, pubbliche e private, è tenuto a concorrere tutto il personale, compresi i liberi professionistiche vi operano in regime di convenzione con il Servizio sanitario nazionale."

- $\quad$ art. 2 "Viene attribuita la funzione di garante per il diritto alla salute al Difensore civico regionale o provinciale e istituzione dei centri regionali per la gestione del rischio sanitario e la sicurezza del paziente."

- art.3 Osservatorionazionale delle buone pratiche sulla sicurezza nella sanità.

- $\quad$ art.4 Trasparenza dei dati.

- art.5 Buone pratiche clinico-assistenziali e raccomandazioni previste dalle linee guida.

- art.6 Responsabilità penale dell'esercente la professione sanitaria.

- art.7 Responsabilità civile della struttura e dell'esercente la professione sanitaria.

- $\quad$ art.8 Tentativo obbligatorio di conciliazione.

- $\quad$ art.9 Azione la rivalsa o di responsabilità amministrativa.

- $\quad$ art.10 Obbligo di assicurazione.

- $\quad$ art.11 Estensione della garanzia assicurativa.

- $\quad$ art.12 Azione diretta del soggetto danneggiato.

- art.13 Obbligo di comunicazione all'esercente la professione sanitaria del giudizio basato sulla sua responsabilità.

- $\quad$ art.14 Fondo di garanzia per danni derivanti da responsabilità sanitaria.

- $\quad$ art.15 Nomina dei consulenti tecnici d'ufficio e dei periti nei giudizi di responsabilità sanitaria.

\section{PREVENZIONE ORGANIZZATIVA} PUNTI DI FORZA

È buona norma in un Dipartimento di Radiologia approntare un registro dove segnalare eventuali cause di errori e malfunzionamento apparecchiature e di monitorare, con riunioni mensili del personale paramedico operante nel Servizio di Radiologia per Immagini, le cause degli eventi sentinella, evenienze che si manifestano in modo assolutamente inatteso ma che costituiscono momenti rivelatori di gravi criticità del sistema, che possono portare l'operatore ad incorrere in eventuali errori professionali.

Ora affrontiamo il management risk in maniera pratica, esaminando alcuni casi di errore in un servizio di Radiologia per Immagini delineando dapprima la figura del coordinatore di un servizio di Radiologia. In primis si devono considerare gli errori Organizzativi; essi sono prettamente di responsabilità del Coordinatore. Le responsabilità non sono esclusivamente del dipendente, sono soprattutto di tipo verticistico-piramidale. Nel caso in cui vi sia un errore da parte di un dipendente, la giurisprudenza chiederebbe al Dirigente se l'errore commesso sia una concausa di problematiche organizzative. Ricordiamo che la gestione del personale, secondo le nuove teorie di organizzazione aziendale, si articola in alcuni istituti fondamentali:

- L'inserimento operativo del dipendente nell'attività lavorativa

- La scelta del personale Front-Line

- La gestione partecipativa e la delega di funzioni 
- La soluzione dei conflitti interni ed esterni

- La formazione permanente del personale

- La sicurezza degli operatori

- Il sistema premiante

Pertanto la gestione del personale non può più essere affidata a Coordinatori professionisti non preparati; essi devono possedere una cultura a 360 gradi, devono prevedere le criticità e gestire i punti di forza, devono affrontare e prevenire le varie problematiche inerenti le apparecchiature ed il personale sotto la propria responsabilità gestendo i rapporti in maniera sempre Oggettiva. In definitiva il coordinatore deve essere un Professionista del Management Risk.

Il Coordinatore di Radiologia deve effettuare riunioni periodiche e approntare un verbale dove sono ascritte le azioni programmatiche con il personale a lui assegnato per categoria, monitorare e nel caso vi siano problematiche tra il personale, trovare delle soluzioni con giudizio sempre Oggettivo. Deve inoltre applicare secondo la necessità il Contratto Nazionale del Sistema Sanitario e il Contratto Decentrato Aziendale, in modo che con il suo comportamento sia sempre corretto con il personale operante e non danneggi il personale sia professionalmente e psicologicamente.

Egli deve, per una crescita professionale continua, approntare fra tutto il personale dei piani di corsi di aggiornamento sia interni alla Asl di appartenenza che sia da effettuare fuori Asl; deve motivare e coinvolgere tutte le risorse umane, mediante regole, incentivazioni monetarie e morali, adeguate condizioni di lavoro, appropriati stili di leadership, assumendosi con ciò l'impegno di responsabilizzare ogni unità lavorativa con la partecipazione e l'identificazione nell'azienda.

Il Coordinatore di Radiologia, secondo gli obbiettivi strategici dall'Azienda Sanitaria, deve Organizzare il personale da lui gestito secondo le normative vigenti in merito alla rilevazione dei carichi di lavoro tali da determinare il numero del personale sufficiente per conseguire gli obbiettivi Aziendali e dare la garanzia e sicurezza degli ambienti di lavoro e dei lavoratori, D.lgs.9 aprile 2008, $n^{\circ} 81$. Un valido Coordinatore può essere definito indubbiamente quindi, un punto di forza di un Reparto di Radiologia per Immagini. È di fondamentale importanza che il Coordinatore di Radiologia agisca in piena autonomia professionale.

Ormai la tecnologia in un Dipartimento di Radiologia per le acquisizioni di Immagini Diagnostiche è diventato complesso ed articolato per via dei vari attori che interessati. Ormai da decenni i dati riguardanti i pazienti, l'elaborazione delle immagini e la loro archiviazione sono affidati a sistemi elettronici: il RIS (Radiology Information System) e il PACS (Picture archiving and comunication System) che servono per gestire tutte le attività amministrative e diagnostiche in un unico sistema, dall'accettazione dell'esame alla refertazione, all'archiviazione digitale del referto e delle immagini. Tali sistemi dovrebbero rendere un processo radiologico più semplice e più veloce e produttivo. Nel caso in cui il collega tsrm prestasse la sua opera lavorativa in un Servizio di Radiologia per Immagini, T.C., R.M.N. o in un Servizio di Angiografia o di Sala Operatoria o altre attività di Emergenza attiva operanti nell'arco temporale delle 24 ore lavorative, potrebbe verificarsi un BLOCCO (temporaneo o permanente) dell'apparecchio o del sistema, con conseguenti serie difficolta nel produrre delle Immagini con la loro archiviazione ed elaborazione elettronica impedendo l'esecuzione di esami con acquisizione di Immagini Diagnostiche URGENTI.

Per questa motivazione negli ultimi anni nelle Università Italiane, sono stati istruiti dei corsi Tecnico-Infermieristico con Specializzazione in campo Forense nelle professioni Infermieristiche e Tecniche con lo scopo di determinare a livello Professionale gli errori eseguiti da colleghi tecnici ed infermieri e determinarne una responsabilità penale e risarcitoria.

Il nostro Responsabile del Servizio di Radiologia, a fin di bene, ci incita sempre a trovare delle soluzioni, anche nei casi più disparati e che a noi operatori sembrano, a volte, non avere nessuna soluzione. Le sue parole sono quelle di fare a volte l'impossibile in maniera tale da tutelare il paziente sempre e tutelare il TSRM da eventuali ripercussioni medico legali.

\section{CRITICITÀ}

A questo punto esaminiamo due casi realistici di criticitàche si sono verificati e possono verificarsi in futuro nel Servizio di Radiologia per Immagini. La prima criticità presa in considerazione si occupa del blocco temporale o permanente del sistema di archiviazione RIS e PACS.

Ai colleghi di turno perviene in reparto una richiesta urgente di esami Radiologici di un paziente per determinare la diagnosi (di competenza Medica Radiologica) ed acquisire immagini (di competenza Tecnico Sanitaria Radiologica Medica). Contestualmente per un motivo non precisato si verifica un blocco del sistema RIS/PACS, in uno spazio temporale non precisato; non è pertanto possibile effettuare esami Radiologici e di conseguenza non si possono fare Diagnosi. In questo lasso di tempo il paziente arrivato in Urgenza/ Emergenza perde la vita e successivamente, con esame Medico Legale autoptico, si dimostra che in l'assenza di una diagnosi urgente ne abbia causato il decesso. Si evince che è di fondamentale importanza capire le cause del blocco del sistema RIS e PACS per comunicare a chi si occupa di gestire il Sistema Software (con reperibilità h 24) ogni tipo di problematica per trovare una soluzione tempestiva e risolutiva, affinché ogni attore dell'atto Medico acquisisca e si assuma le proprie responsabilità in maniera tale che il Paziente/Utente sia sempre tutelato.

È fondamentale che ogni Azienda Sanitaria Locale applichi tutti principi tutelativi per i pazienti, in maniera che atti negativi non possano avvenire, con la stipula di servizi con aziende o società che si occupino di Sistemi Software che siano sempre all'altezza delle effettive necessita lavorative e disponibili, qualora vi fossero delle problematiche, a risolverle tempestivamente $h$ 24, poiché un Servizio di Radiologia per Immagini è operativo per le Emergenze con il suo personale h 24. In eventi eccezionali, si deve trovare una soluzione: in questo caso è opportuno Bypassare momentaneamente Il RIS ed eseguire l'inserimento dati del Paziente/ Utente direttamente sulla consolle dell'apparecchio RADIOLOGICO di svariato uso: apparecchio portatile, sala operatoria o quant'altro, T.C., R.M.N.

Per quanto riguarda il sistema di archiviazioni d'immagini PACS, la risoluzione della problematica e di fatto più complessa: dopo aver eseguito l'inserimento dei dati del paziente/utente alla console dell'apparecchiatura si effettua l'esame diagnostico con l'elaborazione delle Immagini diagnostiche. Queste immagini diagnostiche acquisite, successivamente devono essere 
trasferite dal computer dell'apparecchio con programma software precedentemente istallato nel computer, su supporto di archiviazione con programma specifico per l'archiviazione su cd/dvd/usb o quant'altro. La refertazione del Medico Radiologo verrà eseguita su Word o altro sistema di scrittura in maniera tale che sia assicurato al paziente una diagnosi con relative Immagini Diagnostiche. Successivamente con il ripristino del sistema si invia il Referto al sistema RIS e le Immagini al PACS.

Esaminiamo un altro caso di Management Risk riguardante il TSRM: un'avaria al sistema iniettore per la somministrazione dell'apparecchio T.C. o un blocco meccanico per sbalzi di tensione che crea una situazione di black out. Se si fosse messi in condizione di non poter eseguire esami strumentali con iniettore per la somministrazione di mezzo di contrasto e si verificasse un'EMERGENZA, se il paziente perdesse la vita e successivamente con esame autoptico si riscontrasse che l'esecuzione dell'esame T.C. con mezzo di contrasto sarebbe stato Diagnosticamente dirimente per la vita del paziente, si riscontrerebbero sicuramente delle ripercussioni medico legali anche per il TSRM ,poiché è sua la competenza strumentale dell'Iniettore del mezzo di contrasto. La soluzione in questo caso é la somministrazione in Emergenza di mezzo di contrasto in bolo in via venosa o arteriosa; l'esame Diagnostico non sarebbe perfetto, ma qualitativamente sufficiente da poter superare la momentanea crisi venutasi a creare, in modo da non creare una causa di rischio per il paziente bene primario e la sicurezza lavorativa per il tsrm.

Altra causa di rischio da non sottovalutare è la fase di passaggio del paziente dalla barella al tavolo radiologico o lettino t.c. che può causare traumi anche mortali (come già accaduto). Gli eventi di Rischio in un Reparto di diagnostica per Immagini sono considerevolmente aumentati poiché i carichi di lavoro sono aumentati in maniera esponenziale. Purtroppo non possiamo esaminarli e prevenirli tutti, ma con un buon modello organizzativo di qualità, potremmo almeno conoscerlo per prevenirlo.

\section{OBIETTIVI STRATEGICI}

Gli obiettivi che ci si pone sono i seguenti:

1. Adottare un approccio integrato alla gestione del rischio, favorendo l'integrazione delle politiche aziendali relative a sicurezza, qualità, governo clinico, comunicazione con i pazienti e gli operatori, ristoro dei danni.

2. Sviluppare e diffondere la cultura della sicurezza, non punitiva, aperta all'apprendimento, ce coinvolge gli operatori.

3. Diffondere metodologie e pratiche di buona gestione, come espressione di un diffuso e buon "governo clinico".

4. Favorire la conoscenza empirica dei fenomeni di interesse.

5. Intraprendere azioni per ridurre incidenza e gravi- tà di incidenti ed eventi avversi.

6. Minimizzare le conseguenze economiche e di perdita di fiducia a seguito degli incidenti.

7. Gestire in modo efficace i rapporti con le assicurazioni.

8. Gestire in modo efficace i rapporti con altri enti e organizzazioni che interagiscono con l'organizzazione sanitaria nella gestione del rischio.

\section{CONCLUSIONI}

In questo studio abbiamo analizzato:

- La crescita Professionale dei Tecnici Sanitari di Radiologia Medica.

- Chi sono i professionisti(attori) che concorrono a Prestazione Radiologica.

- Gli ultimi aspetti medico-legali della riforma della responsabilità sanitaria.

- La Prevenzione Organizzativa e le criticità-Management Risk.

La figura professionale di un Tecnico Sanitario di Radiologia Medica è la più affascinante ecomplessa tra le figure presenti nel Sistema Sanitario Nazionale Italiano. Figura Professionale che spesso collabora con altre figure di alta professionalità; Medici Radiologi, Cardiologi, Radiologi di Medicina Nucleare, Medici ortopedici di Sala Operatoria e Chirurgica, infermieri professionali, anteponendo in ogni situazione il benessere del paziente/utente. Abbiamo dimostrato con gli anni di essere una categoria con un percorso Formativo Professionale Universitario: laurea triennale, master di primo e secondo livello, percorsi di studi Universitari ormai quinquennali possono aiutarci ad affrontare le criticità che si presentano. Purtroppo le criticità possono non dipendere dalla preparazione del collega operante ma da criteri organizzativi sia di competenza del Coordinatore che del Direttore di Struttura o addirittura della Dirigenza Generale. Esse possono verificarsi per la mancanza di formazione continua (lifelong learning), per apparecchiature obsolete, per sistemi software non appropriati alle necessità operative, sistemi di fornitura elettrica non adeguati alle necessità Ospedaliere e moltissime altre criticità. Tutte queste criticità devono essere valutate e prese in seria considerazione affinché si possa prevenirle. Il superamento delle criticità può avvenire con riunioni tra responsabili e personale operante per capire effettivamente come affrontarle in maniera pratica ed efficace. La carta ei servizi rappresenta una sorta di patto con i cittadini in quanto prevede il raggiungimento di obbiettivi e standard di qualità nell'ottica di un miglioramento continuo, DPCM 19 maggio 1995. Ormai quello che conta nell'Aziende Sanitarie e specialmente nei Servizi sono i numeri di esami che si effettuano, tale a determinare una contabilità economica-patrimoniale, questo cozza con la sicurezza del paziente, poiché la quantità va a discapito della qualità.

\section{REFERENCES}

1. Gazzetta Ufficiale del $1^{\circ}$ agosto 2000 n.251.

2. Gazzetta Ufficiale del 1 febbraio 2006,n.43.

3. Franco Angeli "Management Sanitario".

4. Decreto balduzzi DL $158 \backslash 12$

5. Legge Gelli $08 \backslash 03 \backslash 2017$

6. Ministero della Salute,Risk Management in sanità. Il problema degli errori,Commissione tecnica sul Rischio,Ministero della Salute,Roma. 